\title{
Disrupting Prefrontal Cortex Prevents Performance Gains from Sensory-Motor Training
}

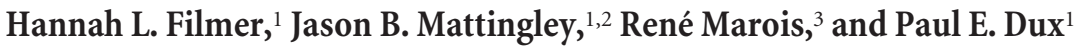 \\ ${ }^{1}$ School of Psychology and ${ }^{2}$ Queensland Brain Institute, The University of Queensland, St Lucia, Queensland 4072, Australia, and ${ }^{3}$ Department of \\ Psychology, Vanderbilt University, Nashville, Tennessee 37235
}

\begin{abstract}
Humans show large and reliable performance impairments when required to make more than one simple decision simultaneously. Such multitasking costs are thought to largely reflect capacity limits in response selection (Welford, 1952; Pashler, 1984, 1994), the information processing stage at which sensory input is mapped to a motor response. Neuroimaging has implicated the left posterior lateral prefrontal cortex (pLPFC) as a key neural substrate of response selection (Dux et al., 2006, 2009; Ivanoff et al., 2009). For example, activity in left pLPFC tracks improvements in response selection efficiency typically observed following training (Dux et al., 2009). To date, however, there has been no causal evidence that pLPFC contributes directly to sensory-motor training effects, or the operations through which training occurs. Moreover, the left hemisphere lateralization of this operation remains controversial (Jiang and Kanwisher, 2003; Sigman and Dehaene, 2008; Verbruggen et al., 2010). We used anodal (excitatory), cathodal (inhibitory), and sham transcranial direct current stimulation (tDCS) to left and right pLPFC and measured participants' performance on high and low response selection load tasks after different amounts of training. Both anodal and cathodal stimulation of the left pLPFC disrupted training effects for the high load condition relative to sham. No disruption was found for the low load and right pLPFC stimulation conditions. The findings implicate the left pLPFC in both response selection and training effects. They also suggest that training improves response selection efficiency by fine-tuning activity in PLPFC relating to sensory-motor translations.
\end{abstract}

\section{Introduction}

Humans can make complex decisions, but under time pressure there are clear capacity limits for even simple choices. A well documented demonstration of these limits is the reaction time (RT) slowing observed in the psychological refractory period paradigm. Here, participants respond more slowly to the second of two simple sensory-motor tasks as the time interval between them decreases (Welford, 1952; Pashler, 1984, 1994). Such multitasking effects are thought to reflect information-processing capacity limits at the relatively elementary decision-making stage of response selection (Welford, 1952; Pashler, 1984, 1994; Dux et al., 2006). Response selection, the mapping of sensory input to a motor response, is a central operation that is amodal both in terms of stimulus and response (Welford, 1952; Pashler, 1984, 1994; Jiang and Kanwisher, 2003; Dux et al., 2006, 2009; Ivanoff et al., 2009).

Neuroimaging research has used both single-task and multitasking paradigms to demonstrate that activity in the left poste-

\footnotetext{
Received May 13, 2013; revised 0ct. 10, 2013; accepted 0ct. 12, 2013.

Author contributions: H.L.F., J.B.M., R.M., and P.E.D. designed research; H.L.F. performed research; H.L.F. analyzed data; H.L.F., J.B.M., and P.E.D. wrote the paper.

This work was supported by an Australian Research Council (ARC) Discovery Grant(DP110102925) to P.E.D. and J.B.M. and the ARC-SRI Science of Learning Research Centre (SR120300015). In addition, P.E.D. was supported by ARC Australian Postdoctoral Fellow (DP0986387) and Future Fellowships (FT120100033) and J.B.M. by an ARC Australian Laureate Fellowship (FL110100103).

Correspondence should be addressed to either Dr. Hannah L. Filmer or Dr. Paul E. Dux, School of Psychology, The University of Queensland, McElwain Building, St Lucia, QLD 4072, Australia, E-mail: h.l.filmer@gmail.com or paul.e.dux@gmail.com.

DOI:10.1523/JNEUROSCI.2019-13.2013

Copyright $\odot 2013$ the authors $\quad 0270-6474 / 13 / 3318654-07 \$ 15.00 / 0$
}

rior lateral prefrontal cortex ( $\mathrm{pLPFC}$ ) correlates with response selection operations (Jiang and Kanwisher, 2003; Dux et al., 2006, 2009; Sigman and Dehaene, 2008; Ivanoff et al., 2009; Hesselmann et al., 2011). For example, the left pLPFC tracks temporal effects associated with multitasking costs and response selection load manipulations (Dux et al., 2006). Moreover, response selection training is associated with a reduction in activity in pLPFC, and a shift in its time course, thought to reflect increased processing efficiency (Dux et al., 2009). Such training effects have been hypothesized to reflect a process of refinement (a reduction in the number, and increase in strength) of neural firing in pLPFC (Dux et al., 2009). To date, however, there has been no direct test of the hypothesis that pLPFC contributes directly to sensory-motor training.

Transcranial direct current stimulation (tDCS) can be used to causally investigate the role of the cortex in behavior. Typically, anodal (excitatory) tDCS improves performance and cathodal (inhibitory) tDCS disrupts performance in motor and cognitive tasks (Nitsche and Paulus, 2000; Nitsche et al., 2003; Utz et al. 2010). Some behaviors are disrupted equally following anodal or cathodal stimulation relative to control measures (Rosenkranz et al., 2000; Ferrucci et al., 2008). The comparison of excitatory and inhibitory stimulation can elucidate the neural mechanisms underlying mental processes. If an operation involves a finely tuned neural response, then either increasing or suppressing activity should impair performance. Alternatively, if an operation requires sufficient numbers of neurons to be excitable, or is affected by background neural noise, excitatory and inhibitory stimulation should lead to dissociable effects on performance. 
A

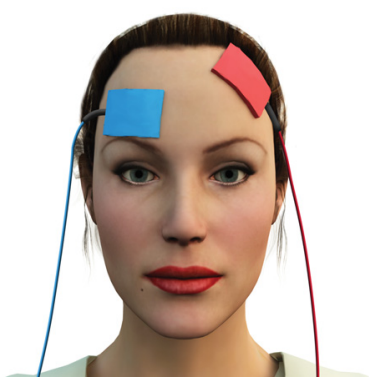

B

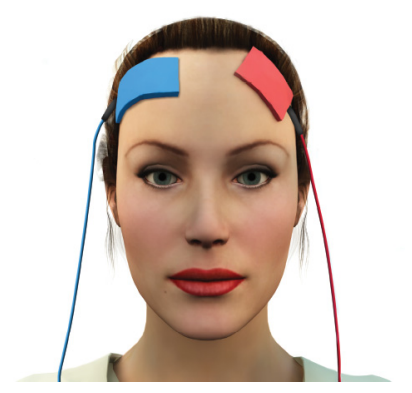

C

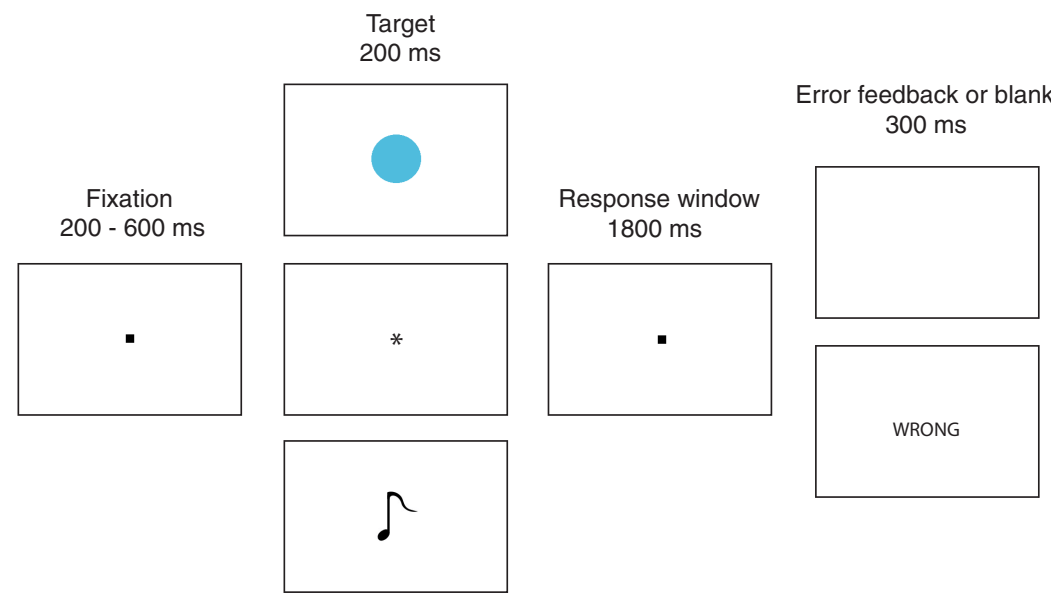

D

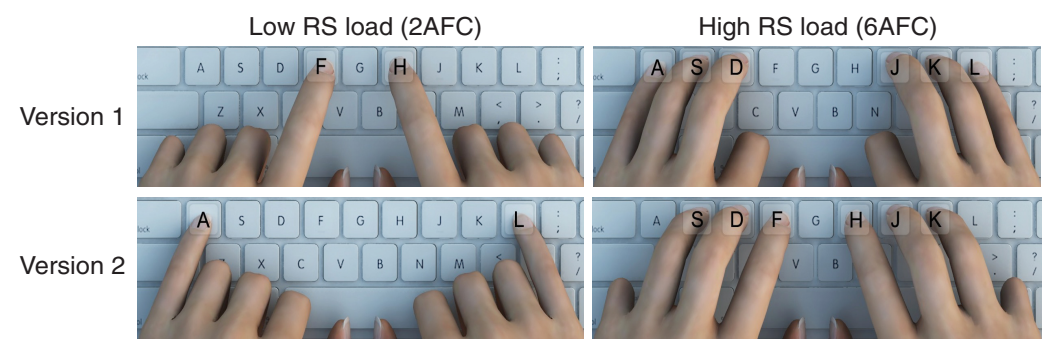

Figure 1. Experiment design. $\boldsymbol{A}, \mathrm{tDCS}$ electrode montage. The target electrode was placed $1 \mathrm{~cm}$ posterior to $\mathrm{F} 3$ (left hemisphere) or F4 (right hemisphere), located with reference to the 10-20 EEG system (Jasper, 1958). The reference electrode was placed over the contralateral orbitofrontal cortex. $\boldsymbol{B}, \mathrm{tDCS}$ electrode montage for the control experiment. The target electrode was placed $1 \mathrm{~cm}$ posterior to $\mathrm{F} 3$ (left hemisphere) and the reference electrode was placed $1 \mathrm{~cm}$ posterior to $\mathrm{F} 4$ (right hemisphere). The reference electrode was larger than the target electrode $\left(35 \mathrm{~cm}^{2}\right)$. C, Standard trial outline. Participants were shown a fixation dot centrally on a monitor, followed by a stimulus (a colored circle, a symbol, or a sound depending on the experimental session), and were instructed to respond as quickly and accurately as they could to the stimulus. $\boldsymbol{D}$, Response configurations for low and high response selection (RS) loads. Participants pressed one of eight keys to make a response, with two keys used for the low RS load blocks, and six keys used for the high RS load blocks. There were two different finger response-keyboard mapping versions, with half of the participants allocated version one and the other half version two.

We examined the role of the left and right pLPFC in response selection and training. Anodal, cathodal, and sham tDCS were applied mid-way through a sensory-motor training protocol. If pLPFC activity becomes more fine-tuned with training (Kelly and Garavan, 2005; Dux et al., 2009), both anodal and cathodal stimulation should disrupt learning. By contrast, if pLPFC is only sensitive to overall excitability or neural noise, differential effects of anodal and cathodal stimulation should be found.

\section{Materials and Methods}

Participants. Eighteen participants, from The University of Queensland community, took part in the left hemisphere experiment $($ mean age $=24$ previous experiments. years, range $=20-33$ years, 13 females), and a different cohort of 18 individuals participated in the right hemisphere experiment (mean age $=23$ years, range $=19-34$ years, $14 \mathrm{fe}$ males). The left hemisphere experiment was run first, and the right hemisphere experiment was run subsequently. All participants had normal (or corrected to normal) vision, and normal hearing. The University of Queensland Human Research Ethics Committee approved the study and all participants gave informed, written consent.

Stimulation protocol. Participants each completed three sessions, with a different type of stimulation used in each (anodal, cathodal, and sham). The order in which the stimulation types were delivered was controlled across participants so that each type of stimulation occurred equally often in each session. The sessions were conducted on average $4 \mathrm{~d}$ apart for both the left- and right-hemisphere groups.

The stimulation was delivered via a NeuroConn stimulator with two $5 \times 5 \mathrm{~cm}$ electrodes. Electrodes were positioned on the scalp with reference to the 10-20 EEG system (Jasper, 1958). The target electrode was placed over the left or right pLPFC, which corresponded to a position $1 \mathrm{~cm}$ posterior to the $\mathrm{F} 3$ site for the left hemisphere, and $1 \mathrm{~cm}$ posterior to the $\mathrm{F} 4$ site for the right hemisphere. Using F3 and F4 as target areas for pLPFC stimulation is common in experiments using tDCS (Utz et al., 2010), and it has previously been confirmed that F3/F4 corresponds to Brodmann area 9 (Herwig et al., 2003), the area most closely related with response selection and the PRP (Dux et al., 2006, 2009). The second electrode was placed over the contralateral supraorbital region (Fig. 1A), which is frequently used as a reference site for prefrontal stimulation (Utz et al., 2010). For the active stimulation conditions (anodal and cathodal), the current was applied for a total of $9 \mathrm{~min}$ (including a $30 \mathrm{~s}$ ramp up/down period at the beginning/end of stimulation) at a current intensity of $0.7 \mathrm{~mA}$. For the sham condition, the parameters were the same but the stimulation lasted for $1 \mathrm{~min} 15 \mathrm{~s}$. Participants were not informed which type of stimulation they were receiving, and sat in silence with their eyes open for the full $9 \mathrm{~min}$ in all three conditions. All participants reported the stimulation was comfortable.

An additional follow-up control experiment was also run with the target electrode over the left pLPFC and a larger reference electrode $(5 \times 7 \mathrm{~cm})$ over the right pLPFC. Using a larger reference electrode reduces the current density at the reference site, minimizing the effect of stimulation at this location. With the stimulation effects for the reference location thus reduced, and a new reference location used that is not involved in response-selection (Dux et al., 2006, 2009; see below), the effect of stimulation on behavior is more directly attributable to the target stimulation region (left pLPFC). Eighteen participants (mean age $=24$ years, range $=20-33$ years, 13 female), who had not taken part in the previous experiments, took part in this control experiment, and the method employed, with the exception of the reference electrode size and placement, was identical to that of the

Behavioral tasks. The experiment used three different tasks in which participants discriminated between colored circles (red: RGB 23732 36, 
dark green: RGB 10130 65, dark blue: RGB 44 71 151, light green: RGB 109205 119, light blue: RGB 79188 220, brown: RGB 167106 48, pink: RGB 25557 255, and yellow: RGB 255 235 30), symbols (\#, \%, @, , ^, *, +, |), or sounds [eight tones, the same as those used by Dux et al. (2006)]. One task was used per experimental session, with the task allocated to each session so participants completed each task only once. Across participants the tasks were paired with each type of stimulation and session (first, second, or third) equally often. Having a new task in each session carried two main advantages. First, each session required new learning, thus allowing for training effects in all three sessions. Second, including tasks involving different modalities and stimuli allowed us to examine whether response selection in the PLPFC is stimulus- and modality-invariant.

For each participant in each session, two of the eight relevant stimuli were randomly selected for the low response selection load condition, and the remaining six were used for the high response selection load condition. Responses were given manually via eight specified keys on a standard Macintosh keyboard (Fig. $1 C$ ), with two fingers/keys used for the 2 alternative forced choice (AFC) blocks (index fingers or little fingers), and the rest used for the 6AFC blocks. Participants were instructed to respond as quickly and accurately as possible.

During the experiment, participants sat 70 cm from a 19" CRT monitor with a refresh rate of $100 \mathrm{~Hz}$. An outline of a standard trial is shown in Figure $1 B$. Before the main experiment began, participants were presented with the stimuli and shown the corresponding response keys so that they could familiarize themselves with the stimulus-response mappings. Two practice blocks of 30 trials for each response selection load condition were then completed to further familiarize participants with the trial structure and give some practice with the response keys. During this period, accuracy feedback was given following each response. Following familiarization, participants trained at the task for 540 trials (without any accuracy feedback), split into three phases for analysis of training effects on performance. This number of trials was selected as previous work has shown the singletask training benefits occur rapidly (Dux et al., 2009). For each phase, participants completed three blocks of 30 trials for each response selection load condition, with the two types of blocks interleaved. Half the participants had the repeating block pattern of high response selection load and then low response selection load, and the other half of the participants had the reverse. Stimulation was applied after the first phase (before tDCS), with the second phase starting immediately after cessation of stimulation (immediate post tDCS), and the last phase starting $20 \mathrm{~min}$ after cessation of stimulation ( $20 \mathrm{~min}$ post $\mathrm{tDCS})$. This design allowed for a measure of performance before stimulation, and a measure of the effect of stimulation on two further training phases. Of primary interest here was how the the difference in behavioral performance between the before $\mathrm{tDCS}$ phase and $20 \mathrm{~min}$ post $\mathrm{tDCS}$ phase was influenced by stimulation, response selection load, and hemisphere as this reflects the conditions under which the greatest effect of training should be observed.

\section{Results}

Repeated-measures ANOVAs were conducted to examine the effects of stimulation type, training phase, and response selection load, separately for the left- and right-hemisphere stimulation experiments (with the orbitofrontal control sites). The
B
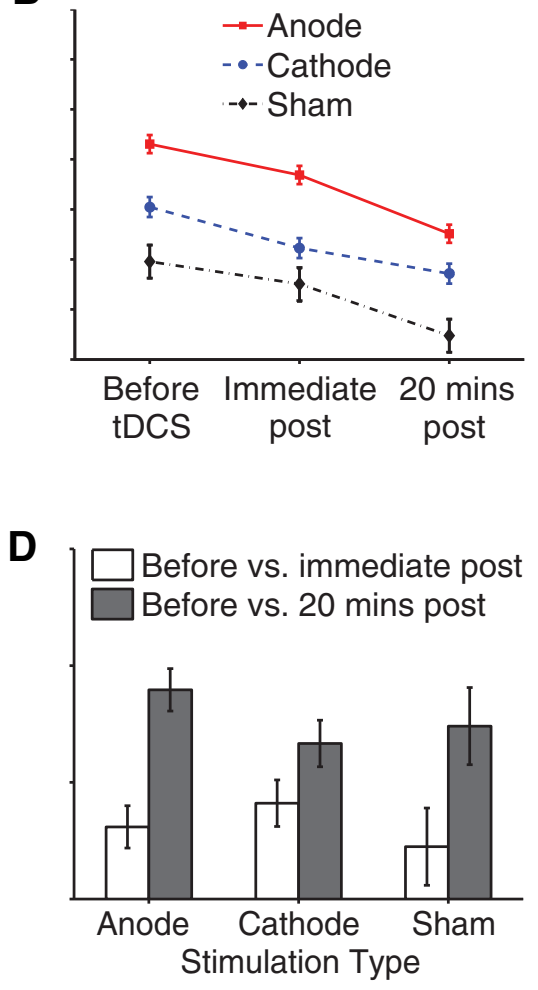

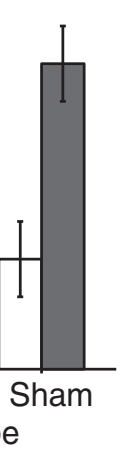

Figure 2. Influence of training and tDCS on the high response selection load condition. $\boldsymbol{A}$ and $\boldsymbol{B}$ show the reaction times (RTs) per stimulation condition for each of the three phases of the experiment for the left and right hemisphere participants, respectively. the left hemisphere reduced with practice, as did RTs for all conditions in the right hemisphere. By contrast, RTs barely reduced with training in conditions involving anodal and cathodal stimulation of the left hemisphere.

RTs for each condition are shown in Figures 2 and 3, and the error data are shown in Table 1. For the left hemisphere RTs, the main interaction of interest-stimulation type $\times$ training phase $\times$ load-was significant $\left(F_{(4,68)}=3.72, p=0.009\right)$. This reflected a training-related reduction in RTs for the sham condition with high response selection load, but no training related reduction in RTs following anodal or cathodal stimulation in either the high load or the low load conditions. In contrast, when the right pLPFC was stimulated, the anodal and cathodal stimulation conditions showed a reduction in the high response selection load RTs with training that was indistinguishable from that observed in the sham condition. Critically, for the right pLPFC conditions, there was no significant interaction between stimulation type, training phase, and response selection load $\left(F_{(4,68)}=0.92, p=0.46\right)$, in contrast to the significant interaction found after left pLPFC stimulation. Importantly, the training effect, i.e., the difference in performance between the before tDCS and 20 min post tDCS phases, interacted significantly with stimulation type, response selection load, and experiment $\left(F_{(2,68)}=\right.$ $3.71, p=0.03)$. Hence, stimulation of the left pLPFC selectively disrupted training-related performance gains relative to stimulation of the right pLPFC. For the error data, there was no significant interaction between stimulation type, training phase, and load for the left hemisphere $\left(F_{(4,68)}=0.73, p=0.57\right)$ or for the right hemisphere $\left(F_{(4,68)}=1.38, p=0.25\right)$. Thus, the effect of stimulation was limited to the RTs and no speed/accuracy tradeoff was observed.

These effects from the omnibus ANOVAs were further examined with follow-up analyses. For stimulation over left hemi- 

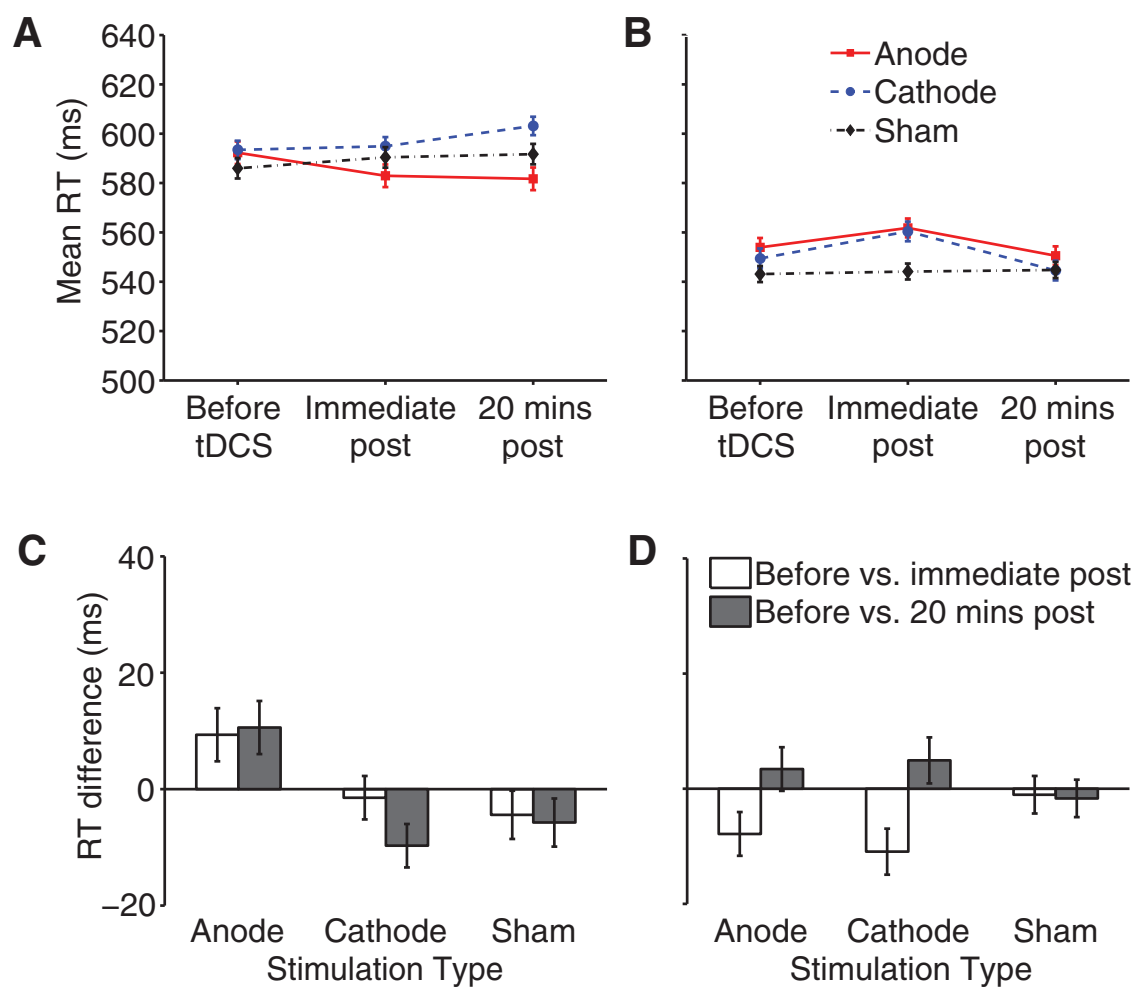

Figure 3. Influence of training and tDCS on the low response selection load condition. $\boldsymbol{A}$ and $\boldsymbol{B}$ show the reaction times (RTs) per stimulation condition for each of the three phases of the experiment for the left and right hemisphere participants, respectively. Error bars represent SEM of the change in RT with training. $\boldsymbol{C}$ and $\boldsymbol{D}$ show the difference in RTs between the before $\mathrm{DCC}$ and the immediate post $\mathrm{tDCS}$ and 20 min post $\mathrm{tDCS}$ phases. The error bars represent the SEM of the change in RT compared with the before tDCS phase. Overall, RTs in all conditions changed little with training, and there were no differences across stimulation protocols.

Table 1. Mean error rates for all conditions

\begin{tabular}{|c|c|c|c|c|c|c|}
\hline & \multicolumn{3}{|c|}{ Left pLPFC } & \multicolumn{3}{|c|}{ Right pLPFC } \\
\hline & $\begin{array}{l}\text { Before } \\
\text { tDCS }\end{array}$ & $\begin{array}{l}\text { Immediate } \\
\text { post tDCS }\end{array}$ & $\begin{array}{l}20 \mathrm{~min} \\
\text { post tDCS }\end{array}$ & $\begin{array}{l}\text { Before } \\
\text { tDCS }\end{array}$ & $\begin{array}{l}\text { Immediate } \\
\text { post tDCS }\end{array}$ & $\begin{array}{l}20 \mathrm{~min} \\
\text { post tDCS }\end{array}$ \\
\hline \multicolumn{7}{|l|}{ Anode } \\
\hline High load & 8.52 & 5.99 & 5.99 & 7.22 & 6.05 & 6.05 \\
\hline Low load & 2.41 & 3.09 & 2.59 & 2.65 & 3.02 & 2.16 \\
\hline \multicolumn{7}{|l|}{ Cathode } \\
\hline High load & 7.59 & 6.67 & 5.93 & 6.67 & 6.23 & 5.93 \\
\hline Low load & 3.46 & 4.26 & 3.27 & 2.84 & 2.35 & 3.52 \\
\hline \multicolumn{7}{|l|}{ Sham } \\
\hline High load & 10.86 & 7.10 & 5.80 & 5.31 & 5.19 & 5.00 \\
\hline Low load & 2.72 & 1.98 & 1.91 & 3.02 & 3.21 & 2.65 \\
\hline
\end{tabular}

The table shows the mean error rates (\%) separately for each stimulation type, experimental phase, and response selection load, and for each hemisphere stimulated.

sphere pLPFC, longer RTs (mean difference $=251 \mathrm{~ms}, \mathrm{SEM}=$ $\left.17, F_{(1,34)}=218.2, p<0.001\right)$ and higher error rates (mean difference $=4.3 \%, F_{(1,34)}=39.8, p<0.001$ ) were found for the high response selection load (mean $\mathrm{RT}=841 \mathrm{~ms}, \mathrm{SEM}=27$, mean error rate $=7.2 \%, \mathrm{SEM}=0.8 \%)$ compared with the low response selection load condition (mean $\mathrm{RT}=590 \mathrm{~ms}, \mathrm{SEM}=$ 22 , mean error rate $=2.9 \%, \mathrm{SEM}=0.4 \%$ ). This confirms that the high response selection load was more difficult than the low response selection load condition, and suggests that our response selection load manipulation tapped the central bottleneck (Dux et al., 2006). ANOVAs run separately for each response selection load condition showed a significant interaction between stimulation type and training phase for the high response selection load condition $\left(F_{(4,68)}=\right.$ $3.58, p=0.01$ ), but not for the low response selection load condition
$\left(F_{(4,68)}=1.19, p=0.32\right)$. The effect of stimulating the left pLPFC was thus specific to the high response selection load condition.

As previously noted, there were clear training-related reductions in RTs for the left and right pLPFC following sham stimulation when the response selection load was high (Fig. 2). The degree of reduction in RTs with training for sham stimulation was somewhat larger for the left than the right pLPFC, but this difference was not significant (difference $=24 \mathrm{~ms}$, $\left.t_{(34)}=1.66\right)$.

There were also numerical differences between the three stimulation conditions in the before tDCS phase for the high response selection load trials. However, these differences were not consistent across the experiments: the main effect of stimulation was not statistically significant across hemispheres for RTs $\left(F_{(2,68)}=0.23\right.$, $p=0.79)$ or errors $\left(F_{(2,68)}=0.28, p=0.74\right)$, and did not significantly interact with experiment for RTs $\left(F_{(2,68)}=0.38, p=0.69\right)$ or errors $\left(F_{(2,68)}=0.34, p=0.69\right)$. There were also no significant overall differences for the two experiment groups in terms of RTs $\left(F_{(1,34)}=1.9, p=0.18\right)$ or errors $\left(F_{(1,34)}=0.41, p=0.53\right)$.

As response selection is thought to be an amodal process (Welford, 1952; Pashler, 1984, 1994; Jiang and Kanwisher, 2003; Dux et al., 2006,2009; Sigman and Dehaene, 2008; Ivanoff et al., 2009), the RT results were examined further by breaking them down into the three different task versions, two of which were visual and one of which was auditory. The results for the high response selection load condition in the left pLPFC showed an identical pattern for all three tasks (Fig. 4). Furthermore, the between-participants factor of task type, when added to the ANOVA for the left pLPFC conditions, did not significantly interact with the factors of stimulation type, training phase, or response selection load $\left(F_{(4,80)}=0.88, p=0.54\right)$. In addition, the interaction between stimulation type, training phase, and response selection load remained significant $\left(F_{(4,60)}=\right.$ $3.66, p=0.01)$ when task type was added to the ANOVA. Thus, the disruption to the training effect found in the averaged data was not driven exclusively by one of the tasks or modalities, but rather was modality invariant.

A follow-up control experiment was conducted to confirm that the right orbitofrontal reference electrode in the first study did not contribute to the reported effects of left pLPFC stimulation on response selection and training. To address this issue in the follow-up experiment, the target electrode was again placed over the left pLPFC, but the reference electrode was placed over right pLPFC (recall that stimulating the right $\mathrm{pLPFC}$ had no effect in the first study). We reasoned that any training-related effect of stimulation in this experiment would confirm a critical role for the left pLPFC, as it would rule out any potential contribution from the right orbitofrontal cortex.

Results from the control experiment are shown in Figure 5. Crucially, we again observed disruption in training for the high response selection load condition-consistent with the first 

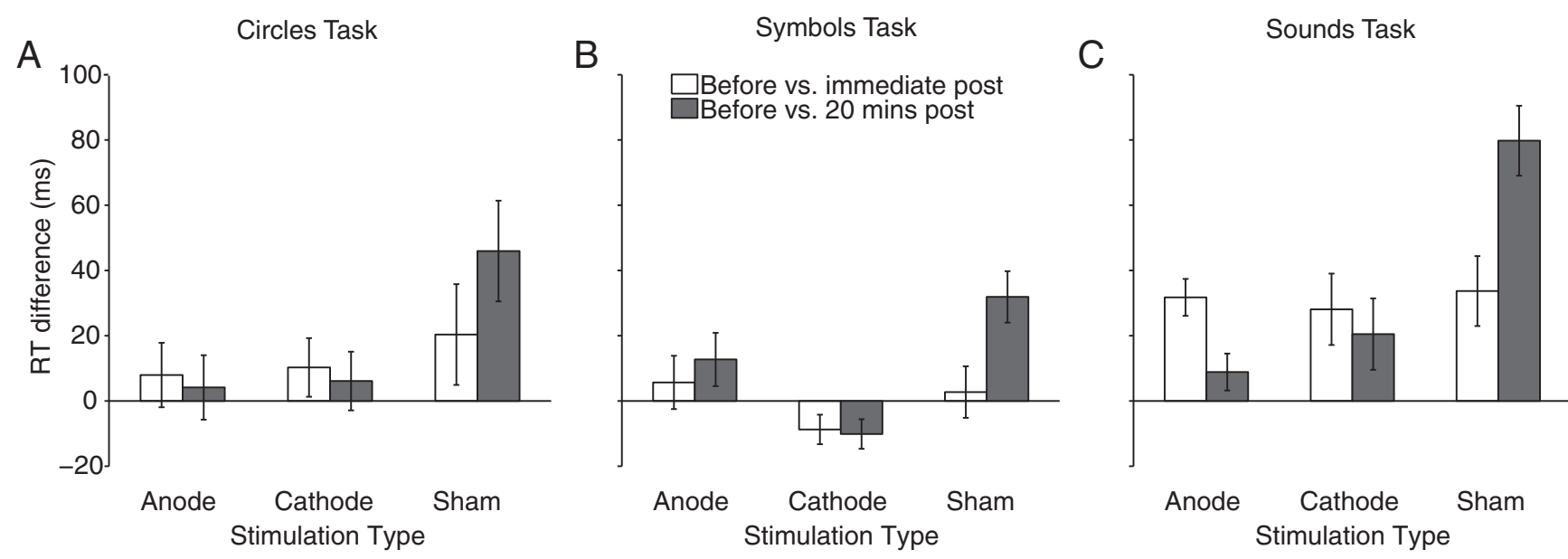

Figure 4. Effect of left hemisphere stimulation on training in the high load condition across the three task types ( $\boldsymbol{A}$, cirlces; $\boldsymbol{B}$, symbols; $\boldsymbol{C}$, sounds). Graphs show the difference in reaction times (RTs) between the immediate post tDCS and 20 min post tDCS phases relative to the before $\mathrm{DCS}$ phase for the left $\mathrm{pLPFC}$. Error bars represent SEM of the change in RT compared with the before $\mathrm{tDCS}$ phase. In all three tasks RTs were reduced from before tDCS to 20 min post tDCS for the sham condition, whereas there was little change in RTs for the active stimulation conditions.

study-following anodal and cathodal stimulation. We had strong a priori predictions for this control experiment, based on the findings of the previous experiment, so planned $t$ tests were used here to analyze the data. The differences in the training effect size for the high response selection load were significant for anodal versus sham stimulation $\left(t_{(17)}=\right.$ $2.74, p=0.01)$, and for cathodal versus sham stimulation $\left(t_{(17)}=2.32, p=0.03\right)$, but not for anodal versus cathodal stimulation $\left(t_{(17)}=0.38, p=0.71\right)$. Moreover, subjecting the data to a full three-way ANOVA, as per the main experiment, with factors of stimulation type, training phase, and response selection load, yielded a marginally significant three-way interaction $\left(F_{(4,68)}=2.35, p=0.07\right)$, confirming the outcomes from the planned comparisons.

To summarize, the control experiment provides a direct replication of the disruption to training observed in the first study, but with a different reference electrode location to rule out any contribution from orbitofrontal cortex.

\section{Discussion}

We sought to determine whether pLPFC contributes directly to sensory-motor training effects by applying tDCS to the left or right pLPFC while participants learned a simple sensory-motor task with one of two different levels of response selection load [6 response alternatives (high) vs 2 response alternatives (low)]. Participants were stimulated with anodal (excitatory), cathodal (inhibitory), or sham tDCS part way through training. Training-related performance gains for the high response selection load condition were disrupted following both anodal and cathodal stimulation of the left pLPFC, relative to performance following sham stimulation. This result was replicated in a control experiment, using an alternative reference elec-
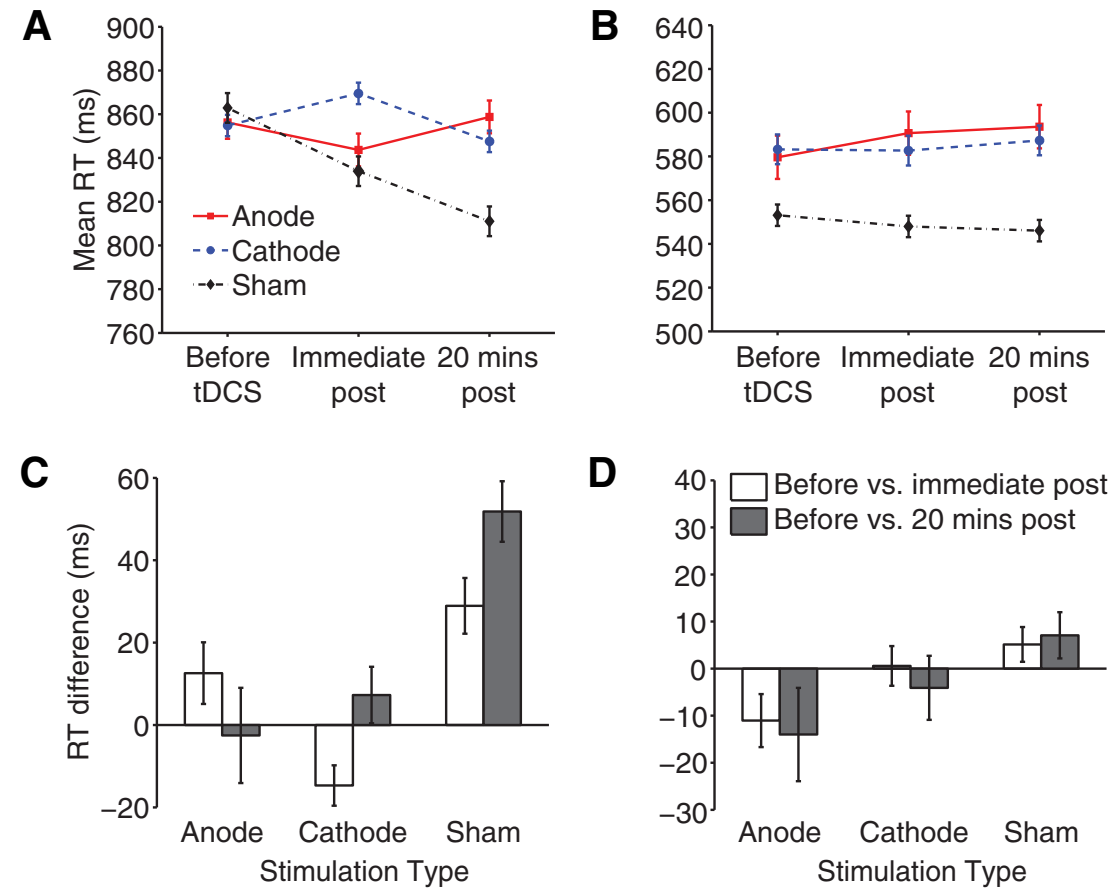

Figure 5. Influence of training and $\mathrm{tDCS}$ on response selection in the control experiment. $\boldsymbol{A}$, Reaction times (RTs) per stimulation condition for each of the three phases of the experiment for the high response selection load condition. Error bars represent SEM of the change in RT with training. $\boldsymbol{B}$, RTs per stimulation condition for each of the three phases of the experiment for the low response selection load condition. Error bars represent SEM of the change in RT with training. $\boldsymbol{C}$, The difference in RTs between the before tDCS and the immediate post tDCS and 20 min post tDCS phases for the high response selection load condition. The error bars represent the SEM of the change in RT compared with the before $\mathrm{DDCS}$ phase. RTs in the sham condition reduced with practice. By contrast, RTs barely reduced with training in conditions involving anodal and cathodal stimulation of the left hemisphere. $D$, The difference in RTs between the before $\mathrm{DDCS}$ and the immediate post $\mathrm{tDCS}$ and 20 min post $\mathrm{tDCS}$ phases for the low response selection load condition. The error bars represent SEM of the change in RT compared with the before $\mathrm{DCS}$ phase.

trode location, confirming the results were due to left pLPFC stimulation. There was no disruption to performance for the lowresponse selection load condition, or to performance for the high- and low-response selection conditions following stimulation of the right pLPFC. Under a low response selection load condition, no effect of stimulation was expected as processing would likely be efficient and trained very quickly given the relatively easy nature of the task. The findings provide the first causal 
evidence that left pLPFC plays a key role in learning sensorymotor mappings.

It is worth noting that there was some variation in performance before stimulation was applied across the stimulation conditions. This variability was not consistent across hemispheres, however, and was not statistically significant; nor did it affect the pattern of behavioral improvement following training. Thus, the apparent before tDCS differences between conditions simply reflect interparticipant noise, and do not alter the significant effects of stimulation observed. The differences were also not apparent in the control experiment, which replicated the effect found in the main left hemisphere experiment. There was also a small difference in the extent of training-related performance gains in the sham condition for the left and right hemispheres. Again, these differences were not significant and likely reflect individual differences across participants.

Together, the findings for the left pLPFC conditions imply modification specifically to response selection processes. An important feature of response selection is its apparent central, stimulus- and response-amodal properties. We used both visual and auditory tasks to test whether the effect of stimulation was stimulus modality-specific, and found the effects were equivalent across modalities. There were some differences in the magnitude of the training effect, and in the reduction in training following tDCS, but the same pattern was present for all three tasks. Also, the allocation of task to stimulation type had no significant effect on the results. This provides evidence, in addition to our load manipulation, that the process we disrupted was indeed response selection, and not disruption of either visual or auditory processing alone. Any explanation for our findings in terms of altered sensory or motor processing is also highly unlikely given that no disruption to performance was found for the low response selection load task, which required visual, auditory, and motor processing.

Two alternative explanations for our results, neither of which involves response selection, must also be considered. First, it might be argued that the stimulation influenced response criterion, i.e., the balance between speed and accuracy, rather than response selection per se. This is highly unlikely, however, given there was no hint of a significant change in error rates with stimulation. Second, it might be argued that stimulation disrupted response maintenance in working memory instead of response selection. This point cannot be ruled out based on the data reported here, although it is improbable as a working memory account would likely affect a change in accuracy, which we did not find. It is also unlikely given previous research into response selection that has explicitly shown that maintenance is not the source of response selection effects (Sigman and Dehaene, 2005; Dux et al., 2006). In addition, at a conceptual level, it is possible that the process of maintaining response mappings in working memory for a pending decision is not distinct from response selection but rather is an integral part of this operation.

We have concluded that our stimulation protocol selectively affected the pLPFC. While there is limited evidence regarding the spatial specificity of tDCS, previous research has reported relatively focal effects of tDCS using $5 \times 5 \mathrm{~cm}$ electrodes (Uy and Ridding, 2003), and it is reasonable to assume that the focal specificity of the current is approximately the same as the electrode size (Rush and Driscoll, 1968). Thus, given we targeted the pLPFC with $5 \times 5 \mathrm{~cm}$ electrodes, the region we affected certainly encompassed the pLPFC, although it is possible the stimulation extended more broadly to other areas of the LPFC.
Previous research has used brain imaging methods to examine the neural substrates of response selection and training (Jiang and Kanwisher, 2003; Dux et al., 2006, 2009; Marois et al., 2006; Sigman and Dehaene, 2008; Ivanoff et al., 2009; Hesselmann et al., 2011), including the initial learning of new arbitrary stimulus-response mappings (Brovelli et al., 2008; Ruge and Wolfensteller, 2010). Our findings provide strong evidence that the left pLPFC plays a causal role in both response selection and response selection training. Furthermore, as both the excitatory (anodal) and inhibitory (cathodal) stimulation protocols disrupted training, we can infer something about the manner in which the left pLPFC regulates performance gains with training. Our data imply that neural ensembles subserving sensory-motor translation within the left pLPFC become increasingly fine-tuned or "sharpened" with training. Any disruption to this system - in either excitatory or inhibitory terms - impairs the task exposure-related increase in neural efficiency that gives rise to sensory-motor training effects. This is in agreement with the hypothesis that neurons selective for response selection coding become more involved with task exposure, and functionally "drop out" if they are not involved in, or do not strongly code for, amodal response selection (Dux et al., 2009).

The nonspecific effect of left anodal and cathodal stimulation we observed is in contrast to many tDCS studies that have described polarity-specific effects of tDCS on motor and cognitive tasks (Nitsche and Paulus, 2000; Nitsche et al., 2003, 2007; Galea et al., 2009; Sparing et al., 2009; Utz et al., 2010; Jacobson et al., 2011). Interestingly, however, research specifically aimed at examining the effect of cortical stimulation on training has typically revealed nonspecific effects of stimulation. For example, both anodal and cathodal stimulation of the cerebellum can disrupt training-related performance gains in working memory (Ferrucci et al., 2008). In addition, when either anodal or cathodal stimulation are applied to the motor cortex, performance gains following training of specific movements can be impaired (Rosenkranz et al., 2000). Our results are thus broadly consistent with this literature in showing polarity nonspecific disruption of training for sensory-motor decision-making after left pLPFC stimulation. A tentative hypothesis is that some training-related performance gains for a range of behaviors could represent a process of neural-sharpening in relevant brain areas.

The current results also suggest a left lateralization of response selection in PLPFC, in line with previous neuroimaging findings (Dux et al., 2006; Dux et al., 2009). By contrast, the left lateralization is inconsistent with evidence for bilateral involvement of frontal cortex in response selection (Jiang and Kanwisher, 2003; Sigman and Dehaene, 2008; Ivanoff et al., 2009), although activations in the left hemisphere tend to cover a larger area and be more robust (Ivanoff et al., 2009). Moreover, there is both correlational (Levy and Wagner, 2011) and causal (Chambers et al., 2007; Verbruggen et al., 2010; Jacobson et al., 2011) evidence to suggest the right pLPFC (a more ventral area of the pLPFC than usually reported in response selection studies) is critically involved in response inhibition, a cognitive operation argued to be related to response selection (Mostofsky and Simmonds, 2008). Our findings clarify this debate by providing compelling causal evidence for the left lateralization of response selection within the pLPFC. Thus, it appears the left pLPFC is predominantly involved in response selection, whereas the right pLPFC may be responsible for response inhibition.

In conclusion, our findings provide causal evidence that the left hemisphere pLPFC plays a key role in response selection and response selection training. Training effects are not observed when processing in this region is impaired. We found that both 
excitatory (anodal) and inhibitory (cathodal) stimulation produced a marked disruption of response selection training. This polarity-nonspecific stimulation effect on training-related performance gains in RTs provides the first direct evidence that the left pLPFC becomes increasingly fine-tuned and efficient for sensory-motor translation with training.

\section{References}

Brovelli A, Laksiri N, Nazarian B, Meunier M, Boussaoud D (2008) Understanding the neural computations of arbitrary visuomotor learning through fMRI and associative learning theory. Cereb Cortex 18:14851495. CrossRef Medline

Chambers CD, Bellgrove MA, Gould IC, English T, Garavan H, McNaught E, Kamke M, Mattingley JB (2007) Dissociable mechanisms of cognitive control in prefrontal and premotor cortex. J Neurophysiol 98:3638-3647. CrossRef Medline

Dux PE, Ivanoff J, Asplund CL, Marois R (2006) Isolation of a central bottleneck of information processing with time-resolved fMRI. Neuron 52: 1109-1120. CrossRef Medline

Dux PE, Tombu MN, Harrison S, Rogers BP, Tong F, Marois R (2009) Training improves multitasking performance by increasing the speed of information processing in human prefrontal cortex. Neuron 63:127-138. CrossRef Medline

Ferrucci R, Marceglia S, Vergari M, Cogiamanian F, Mrakic-Sposta S, Mameli F, Zago S, Barbieri S, Priori A (2008) Cerebellar transcranial direct current stimulation impairs the practice-dependent proficiency increase in working memory. J Cogn Neurosci 20:1687-1697. CrossRef Medline

Franz VH, Loftus GR (2012) Standard errors and confidence intervals in within-subjects designs: generalizing Loftus and Masson (1994) and avoiding the biases of alternative accounts. Psychon Bull Rev 19:395-404. CrossRef Medline

Galea JM, Jayaram G, Ajagbe L, Celnik P (2009) Modulation of cerebellar excitability by polarity-specific noninvasive direct current stimulation. J Neurosci 29: 9115-9122. CrossRef Medline

Herwig U, Satrapi P, Schönfeldt-Lecuona C (2003) Using the international 10-20 EEG system for positioning of transcranial magnetic stimulation. Brain Topogr 16:95-99. CrossRef Medline

Hesselmann G, Flandin G, Dehaene S (2011) Probing the cortical network underlying the psychological refractory period: a combined EEG-fMRI study. Neuroimage 56:1608-1621. CrossRef Medline

Ivanoff J, Branning P, Marois R (2009) Mapping the pathways of information processing from sensation to action in four distinct sensorimotor tasks. Hum Brain Mapp 30:4167-4186. CrossRef Medline

Jacobson L, Javitt DC, Lavidor M (2011) Activation of inhibition: diminishing impulsive behavior by direct current stimulation over the inferior frontal gyrus. J cognitive neuroscience, 23:3380-3387. CrossRef

Jasper HH (1958) The ten-twenty electrode system of the International Federation. Electroencephalogr Clin Neurophysiol 10:5.

Jiang Y, Kanwisher N (2003) Common neural substrates for response selection across modalities and mapping paradigms. J Cogn Neurosci 15: 1080-1094. CrossRef Medline

Kelly AMC, Garavan H (2005) Human functional neuroimaging of brain changes associated with practice. Cereb Cortex 15:1089-1102. Medline

Levy BJ, Wagner AD (2011) Cognitive control and right ventrolateral pre- frontal cortex: reflexive reorienting, motor inhibition, and action updating. Ann N Y Acad Sci 1224:40-62. CrossRef

Marois R, Larson J, Chun M, Shima D (2006) Response-specific sources of dual-task interference in human pre-motor cortex. Psychological Res70:436-447.

Mostofsky SH, Simmonds DJ (2008) Response inhibition and response selection: two sides of the same coin. J Cogn Neurosci 20:751-761. CrossRef Medline

Nitsche MA, Paulus W (2000) Excitability changes induced in the human motor cortex by weak transcranial direct current stimulation. J Physiol 527:633-639. CrossRef Medline

Nitsche MA, Nitsche MS, Klein CC, Tergau F, Rothwell JC, Paulus W (2003) Level of action of cathodal DC polarisation induced inhibition of the human motor cortex. Clin Neurophysiol 114:600-604. CrossRef Medline

Nitsche MA, Doemkes S, Karaköse T, Antal A, Liebetanz D, Lang N, Tergau F, Paulus W (2007) Shaping the effects of transcranial direct current stimulation of the human motor cortex. J Neurophysiol 97:3109-3117. CrossRef Medline

Pashler H (1984) Processing stages in overlapping tasks: evidence for a central bottleneck. J Exp Psychol Hum Percept Perform 10:358-377. CrossRef Medline

Pashler H (1994) Dual-task interference in simple tasks: data and theory. Psychol Bull 116:220-244. Medline

Rosenkranz K, Nitsche MA, Tergau F, Paulus W (2000) Diminution of training-induced transient motor cortex plasticity by weak transcranial direct current stimulation in the human. Neurosci Lett 296:61-63. CrossRef Medline

Ruge H, Wolfensteller U (2010) Rapid formation of pragmatic rule representations in the human brain during instruction-based learning. Cereb Cortex 20:1656-1667. CrossRef Medline

Rush S, Driscoll DA (1968) Current distribution in the brain from surface electrodes. Anesth Analg 47:717-723. Medline

Sigman M, Dehaene S (2005) Parsing a cognitive task: a characterization of the mind's bottleneck. PLoS Biol 3:e37. CrossRef Medline

Sigman M, Dehaene S (2008) Brain mechanisms of serial and parallel processing during dual-task performance. J Neurosci 28:7585-7598. CrossRef Medline

Sparing R, Thimm M, Hesse MD, Küst J, Karbe H, Fink GR (2009) Bidirectional alterations of interhemispheric parietal balance by non-invasive cortical stimulation. Brain 132:3011-3020. CrossRef Medline

Utz KS, Dimova V, Oppenländer K, Kerkhoff G (2010) Electrified minds: transcranial direct current stimulation (tDCS) and galvanic vestibular stimulation (GVS) as methods of non-invasive brain stimulation in neuropsychology-A review of current data and future implications. Neuropsychologia 48:2789-2810. CrossRef Medline

Uy J, Ridding MC (2003) Increased cortical excitability induced by transcranial DC and peripheral nerve stimulation. J Neurosci Methods 127: 193-197. CrossRef

Verbruggen F, Aron AR, Stevens MA, Chambers CD (2010) Theta burst stimulation dissociates attention and action updating in human inferior frontal cortex. Proc Natl Acad Sci U S A 107:13966-13971. CrossRef Medline

Welford AT (1952) The 'psychological refractory period' and the timing of high-speed performance-a review and a theory. Br J Psychol 43:2-19. CrossRef 